Journal of Advanced Research in Fluid Mechanics and Thermal Sciences

\title{
Boundary Layer Flow, Heat and Mass Transfer of Cu-Water Nanofluid over a Moving Plate with Soret and Dufour Effects: Stability Analysis
}

\author{
Najwa Najib ${ }^{1,}$, , Norfifah Bachok ${ }^{2}$ \\ Center of American Education, INTI International University, Persiaran Perdana BBN, 71800 Nilai, Negeri Sembilan, Malaysia \\ Department of Mathematics and Institute for Mathematical Research, Universiti Putra Malaysia, 43400 UPM Serdang, Selangor, Malaysia
}

ARTICLE INFO ABSTRACT

Article history:

Received 28 August 2020

Received in revised form 2 March 2021

Accepted 5 March 2021

Available online 14 April 2021

Keywords:

Soret and Dufour effects; stability analysis; moving plate; nanofluid

\begin{abstract}
Our main focus in this paper is to investigate the effects of Soret and Dufour known as thermodiffusion and diffusion-thermo on moving plate in copper water nanofluid. The set of partial differential equations are converted into set of ordinary differential equations using the appropriate similarity variables before being solved numerically using bvp4c code in Matlab software. The results of heat and mass transfer, temperature and concentration profiles on Soret as well as Dufour effects are presented graphically. Soret effect increases the heat transfer rate at the surface while Dufour effect decreases the mass transfer rate at the surface. Since the solutions exist in dual, we carry out the stability solutions to determine which solution is stable and hence the physical meaning is realized physically.
\end{abstract}

\section{Introduction}

The consideration of Soret effect (thermodiffusion) and Dufour effect (diffusion-thermo) in some literature has gain an attraction due to their significance of study when density difference existed in the flow regime [1]. Soret effect can be described as diffusion of particles from higher temperature towards lower temperature (temperature gradient) due to mass flux. Dufour effect is a reverse phenomenon of Soret effect where particles are diffused from higher concentration to the lower concentration due to energy flux. Both effects have been set up in crystal growth process where the behaviour of convective flows has a strong effect on the temperature and solute temporal variations which lead to nonuniform crystal growth and undesirable nonhomogeneous crystals [2]. Apart from that, many applications of thermodiffusion in industrial processes such in fabrication of semiconductor devices in molten metal and semiconductor mixtures, separation of polymers and DNA as well as in optimum oil recovery from hydrocarbon reservoirs [3]. Kafoussias and Williams [4] are among the first researchers who discovered the existence and development of Soret and Dufour effects on mixed convection with temperature dependent viscosity. Some literatures on moving plate under consideration of Soret and Dufour effects have been listed in [5-10].

\footnotetext{
* Corresponding author.

E-mail address: najwa.mohdnajib@newinti.edu.my
}

https://doi.org/10.37934/arfmts.82.1.96104 
The purpose of this work is to extend the work by Bachok et al., [11] in the presence of thermodiffusion (Soret effect) and diffusion-thermo (Dufour effect) in copper water nanofluid on a moving plate. The stability analysis is performed due to dual solutions obtained. Therefore, we implemented the pioneer research on stability solutions done by Merkin [12], Weidman et al., [13], and Harris et al., [14]. The consideration on stability solutions can be discovered in the studies by Roşca and Pop [15], Bachok et al., [16], Ismail et al., [17], Najib et al., [18], and Najib et al., [19].

\section{Methodology}

Consider a two-dimensional laminar boundary layer flow on a fixed or continuously moving flat surface in a water-based nanofluid containing copper ( $\mathrm{Cu}$ ) nanoparticles in the presence of Soret and Dufour effects. It is assumed that the plate moves in the same or opposite direction to the free stream, both with constant velocities. The nanoparticles are assumed to have a uniform spherical shape and size. The boundary layer equations are given by Bachok et al., [11], and Balla and Naikoti [2]

$\frac{\partial u}{\partial x}+\frac{\partial v}{\partial y}=0$

$\frac{\partial u}{\partial t}+u \frac{\partial u}{\partial x}+v \frac{\partial u}{\partial y}=\frac{\mu_{n f}}{\rho_{n f}} \frac{\partial^{2} u}{\partial y^{2}}$

$\frac{\partial T}{\partial t}+u \frac{\partial T}{\partial x}+v \frac{\partial T}{\partial y}=\alpha_{n f} \frac{\partial^{2} T}{\partial y^{2}}+\frac{D_{m} K_{T}}{c_{s} c_{p}} \frac{\partial^{2} C}{\partial y^{2}}$

$\frac{\partial C}{\partial t}+u \frac{\partial C}{\partial x}+v \frac{\partial C}{\partial y}=D_{m} \frac{\partial^{2} C}{\partial y^{2}}+\frac{D_{m} K_{T}}{T_{m}} \frac{\partial^{2} T}{\partial y^{2}}$

along with the initial and boundary conditions

$t<0: u=v=0, \quad T=T_{\infty}, \quad C=C_{\infty}$ for any $x, y$

$t \geq 0: u=U_{w}, \quad v=0, \quad T=T_{f}, \quad C=C_{f}$ at $y=0$

$u \rightarrow U_{\infty}, \quad T \rightarrow T_{\infty}, \quad C \rightarrow C_{\infty}$, as $y \rightarrow \infty$.

where $U_{w}$ and $U_{\infty}$ are constants and correspond to the plate velocity and the free stream velocity, respectively. Further, $u$ and $v$ are the velocity components along the $x$ and $y$ directions, respectively. $D_{m}$ is the diffusion coefficient, $K_{T}$ is the thermal diffusion ratio, $T_{m}$ is mean fluid temperature, $c_{p}$ is the specific heat at constant pressure and $c_{S}$ is concentration susceptibility. $T$ is the temperature of the nanofluid, $\mu_{n f}$ is the viscosity of the nanofluid, $\alpha_{n f}$ is the thermal diffusivity of the nanofluid and $\rho_{n f}$ is the density of the nanofluid, which are given by Oztop and Abu-Nada [20]

$$
\begin{aligned}
& \rho_{n f}=(1-\varphi) \rho_{f}+\varphi \rho_{s}, \quad \alpha_{n f}=\frac{k_{n f}}{\left(\rho C_{p}\right)_{n f}}, \quad \mu_{n f}=\frac{\mu_{f}}{(1-\varphi)^{2.5}} \\
& \left(\rho C_{p}\right)_{n f}=(1-\varphi)\left(\rho C_{p}\right)_{f}+\varphi\left(\rho C_{p}\right)_{s}, \quad \frac{k_{n f}}{k_{f}}=\frac{\left(k_{s}+2 k_{f}\right)-2 \varphi\left(k_{f-} k_{s}\right)}{\left(k_{s}+2 k_{f}\right)+\varphi\left(k_{f-} k_{s}\right)}
\end{aligned}
$$

where $\varphi$ is the nanoparticle volume fraction, $\left(\rho C_{p}\right)_{n f}$ is the heat capacity of the nanofluid, $k_{n f}$ is the effective thermal conductivity of the nanofluid and $C_{p}$ is the specific heat at constant pressure, $k_{f}$ 
and $k_{s}$ are the thermal conductivities of the fluid and of the solid fractions, respectively, $\rho_{f}$ and $\rho_{s}$ are the densities of the fluid and of the solid fractions, respectively.

\subsection{Steady-State Equation $\left(\frac{\partial}{\partial t}=0\right)$}

Introducing the following similarity transformation

$\eta=\left(\frac{U}{v_{f} x}\right)^{1 / 2} y, \quad \psi=\left(v_{f} x U\right)^{1 / 2} f(\eta), \quad \theta(\eta)=\frac{T-T_{\infty}}{T_{f}-T_{\infty}}, \quad \phi(\eta)=\frac{C-C_{\infty}}{C_{f}-C_{\infty}}$

where $\eta$ is the similarity variable, where $U$ is the composite velocity defined as $U=U_{w}+U_{\infty}$. This definition of $U$ was first introduced by Afzal et al., [21] and $\psi$ is the stream function defined as $u=$ $\partial \psi / \partial y$ and $v=-\partial \psi / \partial x$, which automatically satisfied Eq. (1). Substituting the similarity variables (7) into Eq. (2) to Eq. (4) we obtain the following ordinary (similarity) differential equations

$\frac{1}{(1-\varphi)^{2.5}\left(1-\varphi+\varphi \rho_{s} / \rho_{f}\right)} f^{\prime \prime \prime}+\frac{1}{2} f f^{\prime \prime}=0$

$\frac{k_{n f}}{k_{f}} \theta^{\prime \prime}+\operatorname{Pr}\left(1-\varphi+\varphi\left(\rho c_{p}\right)_{s} /\left(\rho c_{p}\right)_{f}\right)\left(\frac{1}{2} f \theta^{\prime}+D u \phi^{\prime \prime}\right)=0$

$\phi^{\prime \prime}+S c\left(\frac{1}{2} f \phi^{\prime}+S r \theta^{\prime \prime}\right)=0$

subject to the boundary conditions (5) which become

$f(0)=0, \quad f^{\prime}(0)=\lambda, \quad \theta(0)=1, \quad \phi(0)=1$

$f^{\prime}(\eta) \rightarrow 1-\lambda, \quad \theta(\eta) \rightarrow 0, \quad \phi(\eta) \rightarrow 0$ as $\eta \rightarrow \infty$

In the above equations, primes denote the differentiation with respect to $\eta$. Here $P r$ is the Prandt number, $S c$ is the Schmidt number, $S r$ is the Soret number, $D u$ is the Dufour number and $\lambda$ is the velocity ratio parameter which are defined as

$\operatorname{Pr}=\frac{v_{f}}{\alpha_{f}}, \quad S c=\frac{v_{f}}{D_{m}}, \quad S r=\frac{D_{m} K_{T}}{v_{f} T_{m}} \frac{T_{f}-T_{\infty}}{C_{f}-C_{\infty}}, \quad D u=\frac{D_{m} K_{T}}{v_{f} c_{s} c_{p}} \frac{C_{f}-C_{\infty}}{T_{f}-T_{\infty}}, \quad \lambda=\frac{U_{w}}{U}$

where $\lambda>0$ corresponds to assisting flow and $\lambda<0$ corresponds to reverse flow.

The physical quantities of practical interest are the local skin friction coefficients $C_{f}$, local Nusselt number $N u_{x}$ and local Sherwood number $S h_{x}$ which are defined as

$C_{f}=\frac{\tau_{w}}{\rho_{f} U_{e}^{2}}, \quad N u_{x}=\frac{x q_{w}}{k_{f}\left(T_{f}-T_{\infty}\right)}, \quad S h_{x}=\frac{x q_{m}}{D_{m}\left(C_{f}-C_{\infty}\right)}$

where $\tau_{w}$ is the skin friction or the shear stresses on the stretching/shrinking sheet, $q_{w}$ is the heat flux from the surface of the plate and $q_{m}$ is the mass flux from the surface of the plate, which are given by

$\tau_{w}=\mu_{n f}\left(\frac{\partial u}{\partial y}\right)_{y=0}, \quad q_{w}=-k_{n f}\left(\frac{\partial T}{\partial y}\right)_{y=0}, \quad q_{m}=-D_{m}\left(\frac{\partial C}{\partial y}\right)_{y=0}$, 
Using Eq. (7) in Eq. (13) and Eq. (14), we obtain

$$
\left(R e_{x}\right)^{1 / 2} C_{f}=\frac{1}{(1-\varphi)^{2.5}} f^{\prime \prime}(0), \quad\left(R e_{x}\right)^{-1 / 2} N u_{x}=-\frac{k_{n f}}{k_{f}} \theta^{\prime}(0), \quad\left(R e_{x}\right)^{-1 / 2} S h_{x}=-\phi^{\prime}(0)
$$

where $R e_{x}=U x / v_{f}$ is the local Reynolds number.

\subsection{Stability Analysis}

Weidman et al., [13] and Roşca and Pop [15] have shown that the lower branch solutions are unstable (not realizable physically), while the upper branch solutions are stable (physically realizable). We test these features by considering the unsteady Eq. (2)-(4). Thus, we introduce the new dimensionless time variable $\tau$. The use of $\tau$ is associated with an initial value problem and is consistent with the question of which solution will be obtained in practice (physically realizable). Using the variables $\tau$ and (7), we have

$\eta=\left(\frac{U}{v_{f} x}\right)^{1 / 2} y, \quad \psi=\left(v_{f} x U\right)^{1 / 2} f(\eta, \tau), \quad \theta(\eta, \tau)=\frac{T-T_{\infty}}{T_{f}-T_{\infty}}, \quad \phi(\eta, \tau)=\frac{C-C_{\infty}}{C_{f}-C_{\infty}}, \quad \tau=\frac{U t}{x}$

so that Eq. (2)-(4) can be written as

$\frac{1}{(1-\varphi)^{2.5}\left(1-\varphi+\varphi \rho_{s} / \rho_{f}\right)} \frac{\partial^{3} f}{\partial \eta^{3}}+\left(\frac{1}{2} f-\tau \frac{\partial f}{\partial \tau}\right) \frac{\partial^{2} f}{\partial \eta^{2}}-\left(1-\tau \frac{\partial f}{\partial \eta}\right) \frac{\partial^{2} f}{\partial \eta \partial \tau}=0$

$\frac{k_{n f}}{k_{f}} \frac{1}{\operatorname{Pr}\left(1-\varphi+\varphi\left(\rho c_{p}\right)_{s} /\left(\rho c_{p}\right)_{f}\right)} \frac{\partial^{2} \theta}{\partial \eta^{2}}+\frac{1}{2} f \frac{\partial \theta}{\partial \eta}+D u \frac{\partial^{2} \phi}{\partial \eta^{2}}-\left(1-\tau \frac{\partial f}{\partial \eta}\right) \frac{\partial \theta}{\partial \tau}=0$

$\frac{1}{S c} \frac{\partial^{2} \phi}{\partial \eta^{2}}+\frac{1}{2} f \frac{\partial \phi}{\partial \eta}+S r \frac{\partial^{2} \theta}{\partial \eta^{2}}-\left(1-\tau \frac{\partial f}{\partial \eta}\right) \frac{\partial \phi}{\partial \tau}=0$

subject to the boundary conditions

$$
\begin{aligned}
& f(0, \tau)=0, \quad \frac{\partial f}{\partial \eta}(0, \tau)=\lambda, \quad \theta(0, \tau)=1, \quad \phi(0, \tau)=1 \\
& \frac{\partial f}{\partial \eta}(\eta, \tau) \rightarrow 1-\lambda, \quad \theta(\eta, \tau) \rightarrow 0, \quad \phi(\eta, \tau) \rightarrow 0
\end{aligned}
$$

To determine the stability of the solution $f=f_{0}(\eta), \theta=\theta_{0}(\eta)$ and $\phi=\phi_{0}(\eta)$ satisfying the boundary-value problem (17)-(19), we write $[13,15]$

$f(\eta, \tau)=f_{0}(\eta)+e^{-\gamma t} F(\eta), \theta(\eta, \tau)=\theta_{0}(\eta)+e^{-\gamma t} G(\eta), \phi(\eta, \tau)=\phi_{0}(\eta)+e^{-\gamma t} H(\eta)$,

where $\gamma$ is an unknown eigenvalue parameter, and $F(\eta), \mathrm{G}(\eta)$ and $H(\eta)$ are small relative to $f=$ $f_{0}(\eta), \theta=\theta_{0}(\eta)$ and $\phi=\phi_{0}(\eta)$. Solutions of the eigenvalue problem (17)-(20) give an infinite set of eigenvalues $\gamma_{1}<\gamma_{2}<\gamma_{3} \ldots$; if $\gamma_{1}$ is negative, there is an initial growth of disturbances and the flow is unstable but when $\gamma_{1}$ is positive, there is an initial decay and the flow is stable. Introducing Eq. (21) into Eq. (17)-(20), we get the following linearized problem 
$\frac{1}{(1-\varphi)^{2.5}\left(1-\varphi+\varphi \rho_{s} / \rho_{f}\right)}{F_{0}}^{\prime \prime \prime}+\frac{1}{2} f_{0} F_{0}{ }^{\prime \prime}+\frac{1}{2} f_{0}{ }^{\prime \prime} F_{0}+\gamma{F_{0}}^{\prime}=0$

$\frac{k_{n f}}{k_{f}} G_{0}{ }^{\prime \prime}+\operatorname{Pr}\left(1-\varphi+\varphi\left(\rho c_{p}\right)_{s} /\left(\rho c_{p}\right)_{f}\right)\left(\frac{1}{2} f_{0} G_{0}{ }^{\prime}+\frac{1}{2} F_{0} \theta_{0}{ }^{\prime}+D u H_{0}{ }^{\prime \prime}+\gamma G_{0}\right)=0$

$H_{0}^{\prime \prime}+S c\left(\frac{1}{2} f_{0} H_{0}{ }^{\prime}+\frac{1}{2} F_{0} \phi_{0}{ }^{\prime}+S r G_{0}^{\prime \prime}+\gamma H_{0}\right)=0$

subject to the boundary conditions

$F_{0}(0)=0, \quad F_{0}{ }^{\prime}(0)=0, \quad G_{0}(0)=0, \quad H_{0}(0)=0$,

$F_{0}^{\prime}(\eta) \rightarrow 0, \quad G_{0}(\eta) \rightarrow 0, \quad H_{0}(\eta) \rightarrow 0$ as $\eta \rightarrow \infty$.

It should be mentioned that for particular values of $\lambda, P r, S r, D u, S c$ and $\varphi$ the stability of the corresponding steady flow solution $f_{0}(\eta), \theta_{0}(\eta)$ and $\phi_{0}(\eta)$, is determined by the smallest eigenvalue $\gamma$. According to Harris et al., [14], the range of possible eigenvalues can be determined by relaxing a boundary condition either on $F_{0}(\eta), G_{0}(\eta)$ or $H_{0}(\eta)$. For the present problem, we relax the condition that $F_{0}{ }^{\prime}(\eta) \rightarrow 0$ as $\eta \rightarrow \infty$ and for a fixed value of $\gamma$ we solve the system (22)-(24) subject to Eq. (25) along with the new boundary condition $F_{0}{ }^{\prime \prime}(0)=1$.

\section{Results}

The system of nonlinear ordinary differential Eq. (8)-(10) along with subjected boundary condition (11) have been solved numerically using bvp4c code in Matlab software. Table 1 depicts the comparison of numerical results for $f^{\prime \prime}(0)$ values which clearly in a good agreement with Bachok et al., [11]. Thus, give us confidence that our numerical results and also plotted figures are correct.

\section{Table 1}

Values of $f^{\prime \prime}(0)$ for some values of $\lambda$ when $\varphi=0.1$

\begin{tabular}{lllll}
\hline$\lambda$ & $\begin{array}{l}\text { Bachok et al., [11] } \\
\text { First Solution }\end{array}$ & Second Solution & $\begin{array}{l}\text { Present results } \\
\text { First Solution }\end{array}$ & Second Solution \\
\hline-0.5 & 0.4674 & 0.2009 & 0.46737 & 0.20091 \\
-0.4 & 0.5117 & 0.0979 & 0.51171 & 0.09792 \\
-0.3 & 0.5097 & 0.0431 & 0.50968 & 0.04314 \\
-0.2 & 0.4844 & 0.0134 & 0.48442 & 0.01343 \\
-0.1 & 0.4433 & 0.0012 & 0.44333 & \\
0 & 0.3901 & & 0.39008 & \\
0.5 & 0 & & 0 & \\
1 & -0.5218 & -0.52133 & \\
\hline
\end{tabular}

The effects of Soret and Dufour are presented in Figure 1 and Figure 2. In Figure 1, we have set the value of Dufour effect $D u$ is equal to $0.15(D u=0.15)$ where we only focusing on different values of Soret effects $S r$. The heat transfer is increasing when we increased the values of $S r$ but the different observation has been seen where mass transfer is decreasing as $\mathrm{Sr}$ increased. Soret effect also known as thermal diffusion (thermodiffusion) where the nanoparticles are diffused from higher temperature to the lower temperature due to the mass flux. The different effects on Dufour $D u$ can be seen in Figure 2 where Soret effect is taken to be $0.15(\mathrm{Sr}=0.15)$. However, increasing Dufour effect $D u$ cause to decrease heat and mass transfer. This is because Dufour effect is the reverse phenomenon of Soret effect called as diffusion-thermo. The nanoparticles diffused from higher 
concentration to the lower concentration due to energy (heat) flux. The dual temperature as well as concentration profiles have been illustrated graphically in Figure 3 and Figure 4 to support our numerical results and the Figure 1 and Figure 2. All profiles satisfy the far field boundary conditions (11) by fulfill the behavior of the flow asymptotically. The boundary layer thickness for the second solution is always thicker than the first solution.
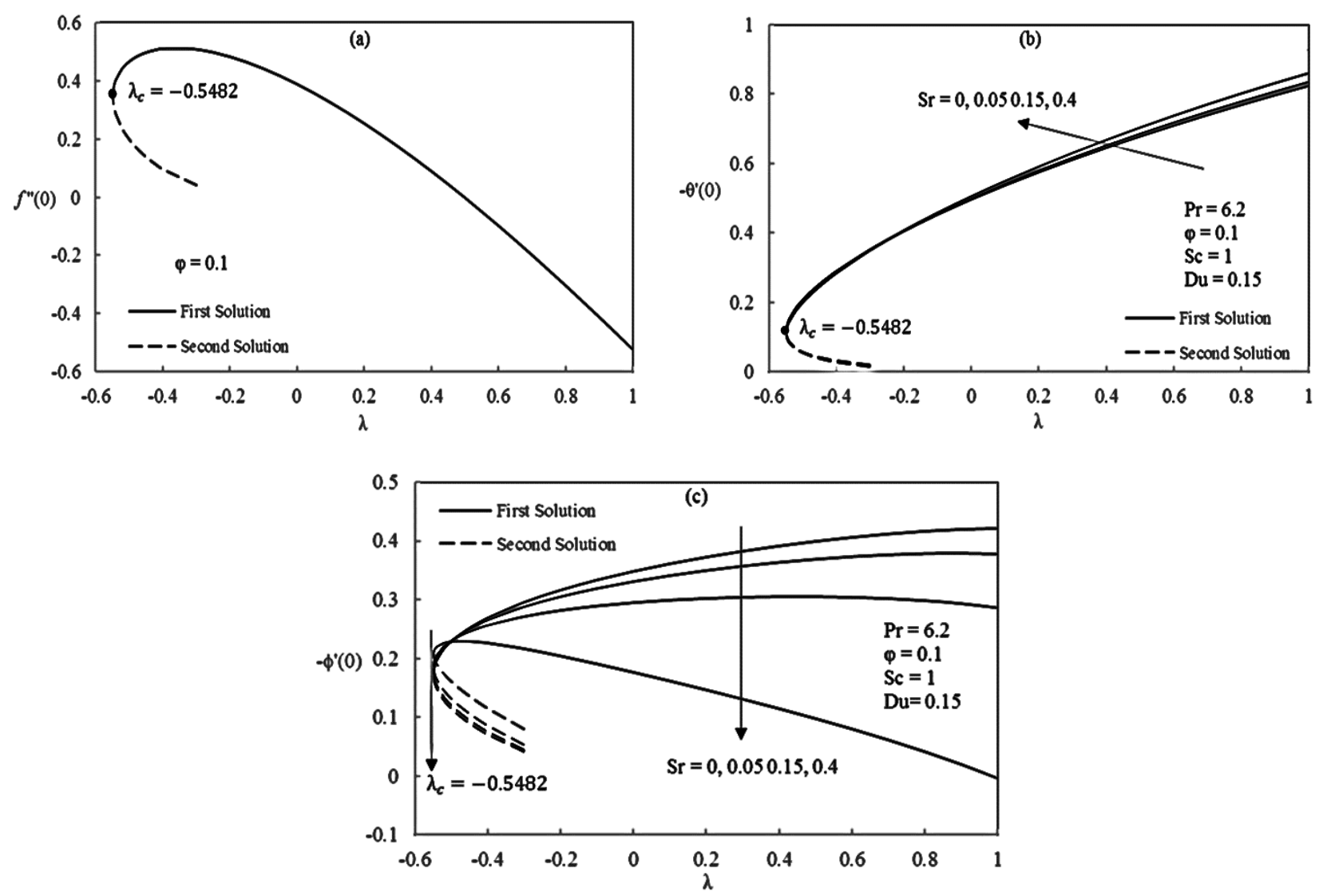

Fig. 1. (a) Skin friction coefficient $f^{\prime \prime}(0)$, (b) temperature gradient $-\theta^{\prime}(0)$ and (c) concentration gradient $-\phi^{\prime}(0)$ vs. $\lambda$ for several values of $S r$
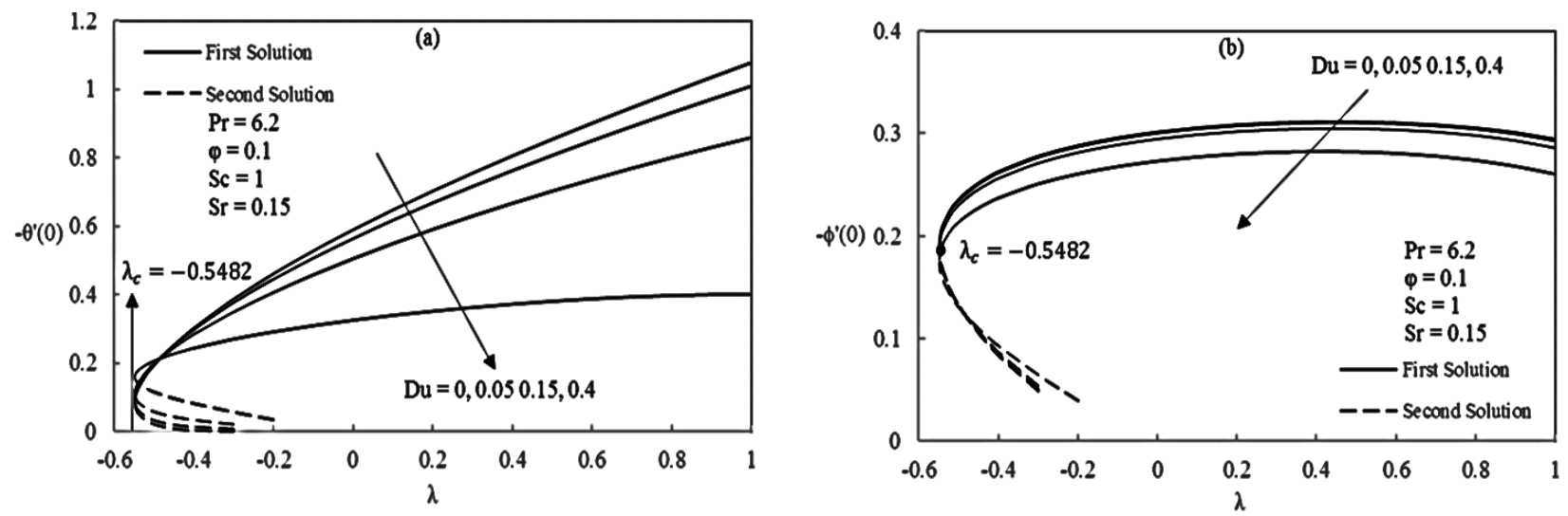

Fig. 2. (a) Temperature gradient $-\theta^{\prime}(0)$ and (b) concentration gradient $-\phi^{\prime}(0)$ vs. $\lambda$ for some values of $D u$

The system of linearized problem (22)-(24) along with the new boundary condition (25) have been applied into code 3 of bvp4c to perform the stability solutions. The smallest eigenvalues $\gamma$ for some values of $\lambda$ are presented in Table 2 . As shown, the eigenvalue $\gamma$ will approaching zero $(\gamma \rightarrow 0)$ when the selected value $\lambda$ is nearer to the critical point $\lambda_{c}$. Moreover, comparison of numerical result with 
previous research indicated that our smallest eigenvalues $\gamma$ are in excellent agreement as stated by Bachok et al., [16]. From our observation, $\gamma$ is positive (stable solution) for the first solution and negative (unstable solution) for the second solution. The solution is said as a stable solution when there only slight disturbance on the flow system that does not affect the flow characteristics while the unstable solution is stated when there existed initial growth of disturbance that affect the flow system. Thus, the first solution is stable and hence they can be realized physically whereas the second solution is not.
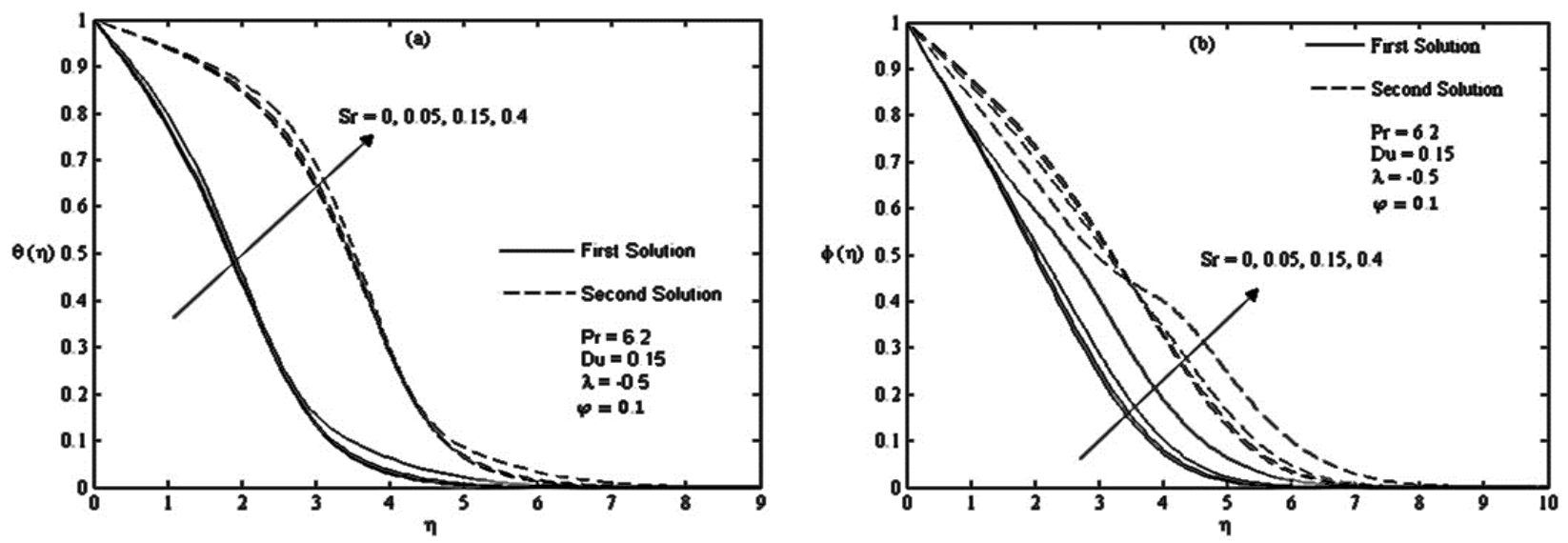

Fig. 3. (a) Dual temperature profile $\theta(\eta)$ and (b) concentration profile $\phi(\eta)$ for some values of $S r$
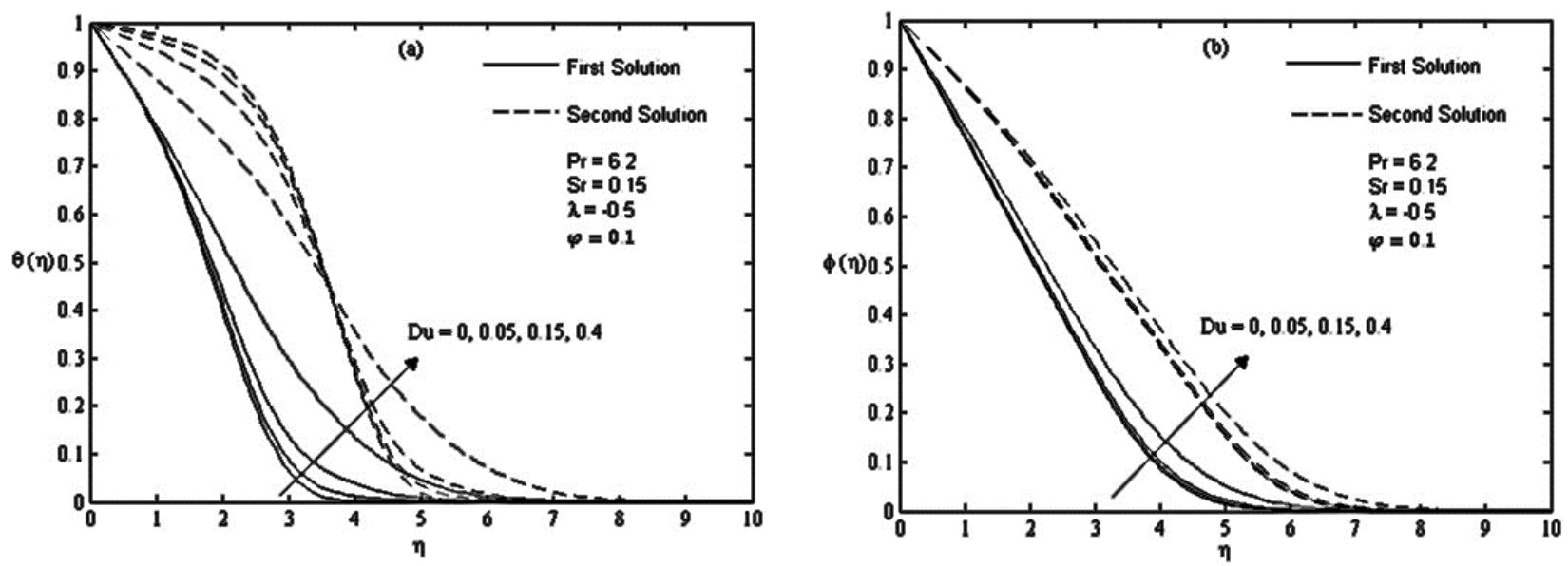

Fig. 4. (a) Dual temperature profile $\theta(\eta)$ and (b) concentration profile $\phi(\eta)$ for some values of $D u$

Table 2

Smallest eigenvalues $\gamma$ for selected values of $\lambda$ when $\varphi=0.1$

\begin{tabular}{|c|c|c|c|c|}
\hline \multirow[t]{2}{*}{$\lambda$} & \multicolumn{2}{|c|}{ Bachok et al., [16] } & \multicolumn{2}{|l|}{ Present results } \\
\hline & First Solution & Second Solution & First Solution & Second Solution \\
\hline-0.5482 & & & 0.0029 & -0.0028 \\
\hline-0.548 & & & 0.0066 & -0.0065 \\
\hline-0.54 & 0.0406 & -0.0350 & 0.0406 & -0.0350 \\
\hline-0.52 & 0.0788 & -0.0597 & 0.0788 & -0.0597 \\
\hline-0.5 & 0.1059 & -0.0733 & 0.1059 & -0.0733 \\
\hline-0.4 & 0.1992 & -0.0982 & 0.1992 & -0.0982 \\
\hline-0.3 & 0.2662 & -0.0949 & 0.2662 & -0.0955 \\
\hline
\end{tabular}




\section{Conclusions}

The effects of Soret and Dufour on boundary layer flow, heat and mass transfer over a moving surface in nanofluid is investigated numerically. The results revealed that

- dual solutions exist only for opposing flow (when the plate and free stream move in opposite direction to each other), $\lambda<0$.

- the first solution is stable and physically realizable while the second solution is not.

- largest $S r$ is required to increase the heat transfer coefficient.

- smallest $D u$ is sufficient to increase heat transfer coefficient.

\section{Acknowledgement}

An express thankfulness to the Universiti Putra Malaysia (Project code: FRGS/1/2018/STG06/UPM/02/4) in the financial support received.

\section{References}

[1] Omowaye, A. J., A. I. Fagbade, and A. O. Ajayi. "Dufour and soret effects on steady MHD convective flow of a fluid in a porous medium with temperature dependent viscosity: Homotopy analysis approach." Journal of the Nigerian Mathematical Society 34, no. 3 (2015): 343-360. https://doi.org/10.1016/i.jnnms.2015.08.001

[2] Balla, Chandra Shekar, and Kishan Naikoti. "Soret and Dufour effects on free convective heat and solute transfer in fluid saturated inclined porous cavity." Engineering Science and Technology, an International Journal 18, no. 4 (2015): 543-554. https://doi.org/10.1016/i.jestch.2015.04.001

[3] Eslamian, Morteza. "Advances in thermodiffusion and thermophoresis (Soret effect) in liquid mixtures." Frontiers in Heat and Mass Transfer (FHMT) 2, no. 4 (2012). https://doi.org/10.5098/hmt.v2.4.3001

[4] Kafoussias, N. G., and E. W. Williams. "Thermal-diffusion and diffusion-thermo effects on mixed free-forced convective and mass transfer boundary layer flow with temperature dependent viscosity." International Journal of Engineering Science 33, no. 9 (1995): 1369-1384. https://doi.org/10.1016/0020-7225(94)00132-4

[5] Motsa, Sandile, and Stanford Shateyi. "Soret and Dufour Effects on Steady MHD Natural Convection Flow Past a Semi-Infinite Moving Vertical Plate in a Porous Medium with Viscous Dissipation in the Presence of a Chemical Reaction." In Evaporation, Condensation and Heat Transfer. IntechOpen, 2011. https://doi.org/10.5772/19683

[6] Subhakar, M. J., K. Gangadhar, and N. Bhaskar Reddy. "Soret and Dufour effects on MHD convective flow of heat and mass transfer over a moving non-isothermal vertical plate with heat generation/absorption." Advances in Applied Science Research 3, no. 5 (2012): 3165-3184.

[7] El-Kabeir, S. M. M., M. Modathar, and A. M. Rashed. "Soret and dufour effects on heat and mass transfer from a continuously moving plate embedded in porous media with temperature dependent viscosity and thermal conductivity." Journal of Modern Methods in Numerical Mathematics 4, no. 2 (2013): 10-22. https://doi.org/10.20454/jmmnm.2013.562

[8] Lavanya, B., and A. Leela Ratnam. "Dufour and soret effects on steady MHD free convective flow past a vertical porous plate embedded in a porous medium with chemical reaction, radiation heat generation and viscous dissipation." Advances in Applied Science Research 5, no. 1 (2014): 127-142.

[9] Rao, G. Srinivasa, B. Ramana, B. Rami Reddy, and G. Vidyasagar. "Soret and Dufour effects on MHD Boundary layer flow over a Moving Vertical porous plate with suction." International Journal of Emerging Trends in Engineering and Development 2, no. 4 (2014): 215-226.

[10] Alao, F. I., A. I. Fagbade, and B. O. Falodun. "Effects of thermal radiation, Soret and Dufour on an unsteady heat and mass transfer flow of a chemically reacting fluid past a semi-infinite vertical plate with viscous dissipation." Journal of the Nigerian Mathematical Society 35, no. 1 (2016): 142-158. https://doi.org/10.1016/i.jnnms.2016.01.002

[11] Bachok, Norfifah, Anuar Ishak, and loan Pop. "Flow and heat transfer characteristics on a moving plate in a nanofluid." International Journal of Heat and Mass Transfer 55, no. 4 (2012): 642-648. https://doi.org/10.1016/j.ijheatmasstransfer.2011.10.047

[12] Merkin, J. H. "On dual solutions occurring in mixed convection in a porous medium." Journal of engineering Mathematics 20, no. 2 (1986): 171-179. https://doi.org/10.1007/BF00042775

[13] Weidman, P. D., D. G. Kubitschek, and A. M. J. Davis. "The effect of transpiration on self-similar boundary layer flow over moving surfaces." International Journal of Engineering Science 44, no. $11-12$ (2006): $730-737$. https://doi.org/10.1016/i.ijengsci.2006.04.005 
[14] Harris, S. D., D. B. Ingham, and I. Pop. "Mixed convection boundary-layer flow near the stagnation point on a vertical surface in a porous medium: Brinkman model with slip." Transport in Porous Media 77, no. 2 (2009): $267-285$. https://doi.org/10.1007/s11242-008-9309-6

[15] Roşca, Alin V., and loan Pop. "Flow and heat transfer over a vertical permeable stretching/shrinking sheet with a second order slip." International Journal of Heat and Mass Transfer 60 (2013): 355-364. https://doi.org/10.1016/i.ijheatmasstransfer.2012.12.028

[16] Bachok, Norfifah, Najwa Najib, Norihan Md. Arifin, and Norazak Senu. "Stability of dual solutions in boundary layer flow and heat transfer on a moving plate in a copper-water nanofluid with slip effect." WSEAS Transactions on Fluid Mechanics 11 (2016): 151-158.

[17] Ismail, Nurul Syuhada, Norihan Md Arifin, Norfifah Bachok, and Norhasimah Mahiddin. "Stagnation-point flow and heat transfer over an exponentially shrinking sheet: A stability analysis." In AIP Conference Proceedings, vol. 1739, no. 1, p. 020023. AIP Publishing LLC, 2016. https://doi.org/10.1063/1.4952503

[18] Najib, Najwa, Norfifah Bachok, and Norihan Md Arifin. "Stability of dual solutions in boundary layer flow and heat transfer over an exponentially shrinking cylinder." Indian Journal of Science and Technology 9, no. 48 (2016): 1-6. https://doi.org/10.17485/ijst/2016/v9i48/97740

[19] Najib, Najwa, Norfifah Bachok, Norihan Md Arifin, and Norazak Senu. "Boundary layer flow and heat transfer of nanofluids over a moving plate with partial slip and thermal convective boundary condition: Stability analysis." International Journal of Mechanics 11, no. 1 (2017): 18-24.

[20] Oztop, Hakan F., and Eiyad Abu-Nada. "Numerical study of natural convection in partially heated rectangular enclosures filled with nanofluids." International Journal of Heat and Fluid Flow 29, no. 5 (2008): 1326-1336. https://doi.org/10.1016/j.ijheatfluidflow.2008.04.009

[21] Afzal, Noor, A. Badaruddin, and A. A. Elgarvi. "Momentum and heat transport on a continuous flat surface moving in a parallel stream." International Journal of Heat and Mass Transfer 36, no. 13 (1993): 3399-3403. https://doi.org/10.1016/0017-9310(93)90022-X 